\title{
Endoplasmic reticulum stress-regulated CXCR3 pathway mediates inflammation and neuronal injury in acute glaucoma
}

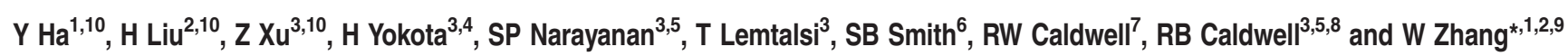

Acute glaucoma is a leading cause of irreversible blindness in East Asia. The mechanisms underlying retinal neuronal injury induced by a sudden rise in intraocular pressure (IOP) remain obscure. Here we demonstrate that the activation of CXCL10/CXCR3 axis, which mediates the recruitment and activation of inflammatory cells, has a critical role in a mouse model of acute glaucoma. The mRNA and protein expression levels of CXCL10 and CXCR3 were significantly increased after IOP-induced retinal ischemia. Blockade of the CXCR3 pathway by deleting CXCR3 gene significantly attenuated ischemic injury-induced upregulation of inflammatory molecules (interleukin-1 $\beta$ and E-selectin), inhibited the recruitment of microglia/monocyte to the superficial retina, reduced peroxynitrite formation, and prevented the loss of neurons within the ganglion cell layer. In contrast, intravitreal delivery of CXCL10 increased leukocyte recruitment and retinal cell apoptosis. Inhibition of endoplasmic reticulum (ER) stress with chemical chaperones partially blocked ischemic injury-induced CXCL10 upregulation, whereas induction of ER stress with tunicamycin enhanced CXCL10 expression in retina and primary retinal ganglion cells. Interestingly, deleting CXCR3 attenuated ER stressinduced retinal cell death. In conclusion, these results indicate that ER stress-medicated activation of CXCL10/CXCR3 pathway has an important role in retinal inflammation and neuronal injury after high IOP-induced ischemia.

Cell Death and Disease (2015) 6, e1900; doi:10.1038/cddis.2015.281; published online 8 October 2015

Acute glaucoma is the major form of glaucoma in East Asia where it is a leading cause of irreversible blindness. ${ }^{1}$ In Western countries, it is less common, but it still has much higher rate to induce vision impairment and blindness than open-angle glaucoma. ${ }^{2}$ Acute glaucoma is a medical emergent condition when intraocular pressure (IOP) is suddenly increased because of blocked drainage canals. ${ }^{1,2}$ Prompt treatment is needed to avoid irreversible glaucomatous optic nerve damage. ${ }^{1}$ Nevertheless, in a substantial portion of patients, acute glaucoma continues progressing to blindness in spite of intensive medical treatment. ${ }^{3} \mathrm{~A}$ rapid rise in IOP that exceeds retinal perfusion pressure is known to cause retinal ischemia and induce retinal neuronal cell death. ${ }^{2,4}$ However, the mechanisms by which elevated IOP induces retinal neuronal injury in acute glaucoma are largely unknown.

Inflammation is the body's defense system against pathogens, ${ }^{5}$ whereas excessive or uncontrolled inflammation induces tissue injury and results in diseases. In the central nervous system (CNS), inflammation has been recognized as a key player in many neurodegenerative diseases, such as
Alzheimer's disease, Parkinson's disease, and Huntington's disease. ${ }^{6}$ Inflammation is involved in the development of glaucoma given that the levels of inflammatory cytokines (e.g., TNF- $a$, interleukin-6, CCL2) and adhesion molecules (e.g., p-selectin) are increased in retina during glaucoma. ${ }^{7,8}$ Moreover, in an animal model of acute glaucoma, inflammatory pathways including toll-like receptors and inflammasome are activated and contribute to retinal neuronal injury.,9 Nonetheless, the key mediators that control inflammatory cell recruitment and activation in this process remain to be elucidated.

Chemokines are a family of pro-inflammatory peptides $(8-15 \mathrm{kD})$ that are produced locally in tissues and mediate leukocyte and microglia directional migration and activation during inflammatory reactions by binding to specific chemokine receptors in the membrane of their respective target cells. CXCR3 is the common receptor for three chemokines belonging to the CXC subclass, namely CXCL9, CXCL10, and CXCL11, ${ }^{10}$ whereas it binds to CXCL4 with low affinity. ${ }^{11-13}$ CXCR3 is critical to mediate the recruitment of activated

\footnotetext{
${ }^{1}$ Department of Ophthalmology and Visual Sciences, The University of Texas Medical Branch, Galveston, TX, USA; ${ }^{2}$ Center for Biomedical Engineering, The University of Texas Medical Branch, Galveston, TX, USA; ${ }^{2}$ Vascular Biology Center, Georgia Regents University, Augusta, GA, USA; ${ }^{4}$ Department of Ophthalmology, Asahikawa Medical University, Asahikawa, Japan; ${ }^{5}$ College of Allied Health Sciences, Georgia Regents University, Augusta, GA, USA; ${ }^{6}$ Cellular Biology and Anatomy, Georgia Regents University, Augusta, GA, USA; ${ }^{7}$ Department of pharmacology and Toxicology, Georgia Regents University, Augusta, GA, USA; ${ }^{8}$ VA Medical Center, Augusta, GA, USA and ${ }^{9}$ Neuroscience and Cell Biology, The University of Texas Medical Branch, Galveston, TX, USA

*Corresponding author: W Zhang, Department of Ophthalmology and Visual Sciences, University of Texas Medical Branch, 301 University Boulevard, Galveston 77555 0144, TX, USA. Tel/Fax: +1 409747 2552; E-mail: we2zhang@utmb.edu

${ }^{10}$ These authors contributed equally to this work.

Abbreviations: AMD, age-related macular degeneration; BM, bone marrow; CNS, central nervous system; ECL, enhanced chemiluminescence; ELISA, enzyme-linked immunosorbent assay; ER, endoplasmic reticulum; GCL, ganglion cell layer; GFP, green fluorescent protein; INL, inner nuclear layer; IOP, intraocular pressure; IR, ischemia-reperfusion; NeuN, neuron-specific nuclear protein; ONL, outer nuclear layer; PBA, 4-phenylbutyric acid; RGC, retinal ganglion cell; TUDCA, tauroursodeoxycholic acid; TUNEL, terminal deoxynucleotidyl transferase-mediated biotinylated UTP nick end labeling; WT, wild-type

Received 29.3.15; revised 18.8.15; accepted 02.9.15; Edited by A Verkhratsky
} 
T cells and microglia/macrophage. ${ }^{10,14-17}$ CXCL10 expression is increased in the central nervous system in neurodegenerative diseases including Alzheimer's disease and multiple sclerosis. ${ }^{18-21}$ The activation of CXCL10/CXCR3 axis has also been shown to promote microglia recruitment and induce neuronal cell death in several models of neurodegeneration. ${ }^{22,23}$ The role of CXCL10/CXCR3 pathway in glaucomatous optic neuropathy is unknown.

In this study, we demonstrate that the CXCL10/CXCR3 axis is activated in a mouse model of acute glaucoma and the activation of this pathway is essential for retinal inflammation and neuronal injury. Moreover, the upregulation of CXCL10 and CXCR3 expression in retina is at least partially mediated by endoplasmic reticulum (ER) stress after ischemia.

\section{Results}

CXCL10 and CXCR3 are upregulated after IOP-induced retinal ischemia. To investigate whether CXCR3 signaling is implicated in acute glaucoma, we examined the expression of CXCR3 and its ligands in a mouse model of acute glaucoma, in which retinal ischemia is induced by acute elevation of IOP. ${ }^{4}$ Analysis of mRNA by quantitative PCR revealed that CXCL10 expression was markedly increased in retinal tissue and reached a peak at $6 \mathrm{~h}$ after ischemia (175-fold of control) (Figure 1a). Consistent with the increase in mRNA, CXCL10 protein was significantly increased as determined by enzyme-linked immunosorbent assay (ELISA) (Figure 1b). CXCL10 mRNA-positive cells were distributed in different retinal layers including cells in the ganglion cell layer (GCL) (Figure 1c). The expression of CXCL4, which binds with low affinity to CXCR3, was elevated after retinal ischemia though expressed later than CXCL10 (Figure 1d). In contrast, the expression of two other ligands, CXCL9, and CXCL11, was undetectable. CXCR3 mRNA was also rapidly upregulated after retinal ischemia (Figure 1e). Immunolocalization analysis showed that immunoreactivity for CXCR3 was present in the inner nuclear layer (INL) and outer nuclear layer in non-injured retina. After ischemic injury, the immunoreactivity for CXCR3 was robustly increased in cells in the GCL (Figure 1f). These data suggest that the CXCL10/ CXCR3 axis may have a role in the pathogenesis of acute glaucoma.

CXCR3 mediates retinal inflammation after ischemic injury. As CXCR3 is expressed in microglia/monocytes, ${ }^{22,24}$ we explored the action of microglia/monocytes after ischemic injury. The distribution of microglia/monocyte, as determined by staining for their specific marker lbal, revealed a prominent increase in the number of Ibal-positive cells in the superficial retina of wild-type (WT) mice after retinal ischemia (Figure 2a) compared with controls. In addition, the Ibalpositive cells were ramified in control retina, whereas many of them in the ischemia-injured retina had phagocytic (ameboid) morphology and were isolectin B4 positive, indicating an activated phenotype. ${ }^{25}$ In contrast, Ibal- and isolectin B4-positive cells were reduced in ischemic retinas of CXCR3-deficient mice and these cells exhibit a significantly less-activated phenotype. In accordance with increased microglia/monocyte recruitment and activation, the expression of CD11b, a marker for microglia/monocyte activation, was also remarkably increased in the retina after ischemic injury, whereas deleting CXCR3 attenuated its upregulation (Figure 2b).

To determine whether lbal-positive cells were derived from retinal local microglia or from monocytes in circulation, we transplanted bone marrow of green fluorescent protein (GFP) mice to WT mice. Our data showed that many Ibal-positive cells were monocytes from the circulation (GFP positive) and some lbal-positive cells were retinal microglia (GFP negative) (Figure 2c), indicating that both microglia and monocytes were recruited to the superficial retina after ischemic injury.

As interleukin-1 $\beta$, a major product of inflammasome, has an important role in inflammation and neurotoxicity in the central nervous system and acute glaucoma, ${ }^{4}$ and E-selectin is involved in the process of leukocyte recruitment by mediating its initial attachment and rolling, ${ }^{26}$ we further determined their expression in ischemia-injured retina. We found that there were significant increases in interleukin-1 $\beta$ and $E$-selectin in WT retinas after ischemic injury, which were markedly blocked by deleting CXCR3 (Figures $2 d$ and e). Together, these data indicate that the activation of CXCR3 pathway mediates microglia/monocyte recruitment and activation and retinal inflammatory reactions after IOP-induced retinal ischemia.

CXCR3 is critically involved in oxidative and nitrosative stress after ischemic injury. During inflammation, local retinal cells and/or recruited leukocytes produce superoxide and nitric oxide, which can not only kill pathogens but also induce tissue injury. To determine whether the activation of CXCR3 is involved in oxidative and nitrosative stress after retinal ischemia, we examined the formation of peroxynitrite in retinal lysates. Peroxynitrite is rapidly formed through the reaction of superoxide and nitric oxide and is an indicator for oxidative and nitrosative stress. Western blot analysis of nitrotyrosine, a marker of peroxynitrite, revealed a prominent increase of peroxynitrite formation in WT retina after ischemic injury. However, this increase was blocked by CXCR3 deletion (Figure 2f). This result suggests that CXCR3 pathway is involved in retinal oxidative and nitrosative stress after IOP-induced retinal ischemia.

CXCR3 pathway has a predominant role in IOP-induced retinal neuronal cell damage. The loss of retinal neurons in the GCL is a hallmark of glaucoma, ${ }^{2,4,27}$ and both inflammation and oxidative stress can cause neuronal cell death; therefore, we investigated whether blocking CXCR3 pathway would protect retinal neuronal cells from IOP-induced cell death. At $24 \mathrm{~h}$ after retinal ischemia, retinal cell apoptosis, as determined by measuring cytoplasmic histone-associated DNA fragmentation using a Cell death ELISA kit, was increased approximately eightfold in ischemia-injured WT retinas. This increase was reduced by $33 \%$ in retinas from mice lacking CXCR3 (Figure 3a). Further analysis of apoptotic cells by terminal deoxynucleotidyl transferasemediated biotinylated UTP nick end labeling (TUNEL) assay revealed that TUNEL-positive (apoptotic) cells were mainly localized in neurons in the GCL and INL at $6 \mathrm{~h}$ after retinal ischemia. At $24 \mathrm{~h}$ after retinal ischemia, apoptotic cells were 


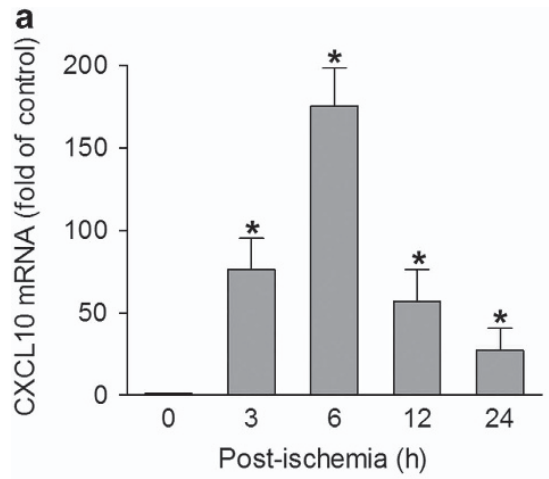

\section{C}
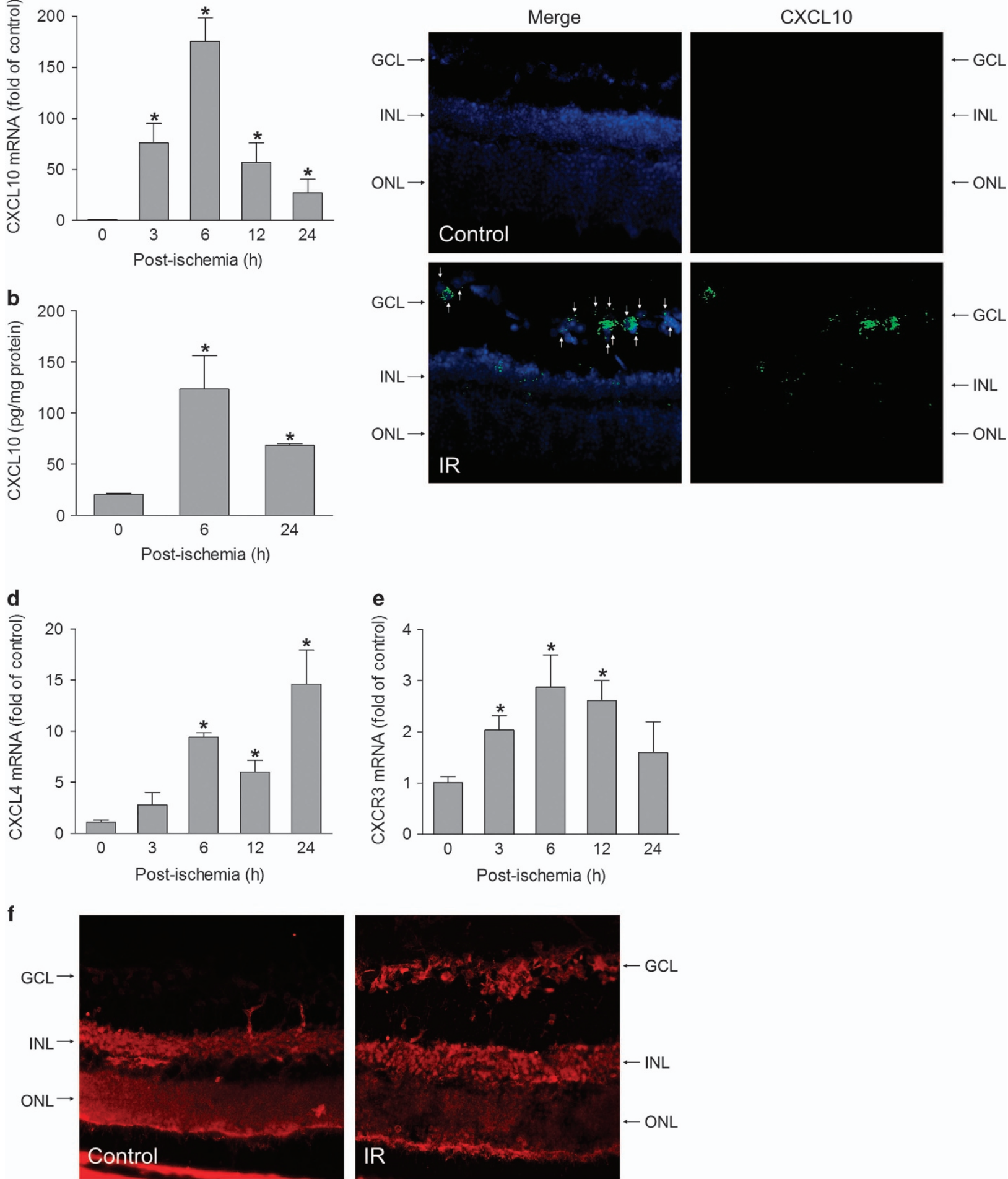

Figure 1 Ischemia-reperfusion induces the expression of CXCR3 and its ligands in retina. Ischemia-reperfusion (IR) was induced in wild-type (WT) mice and retinas were collected at 3, 6, 12, and $24 \mathrm{~h}$ after IR. (a) CXCL10 mRNA was determined by quantitative RT-PCR (qPCR). (b) CXCL10 protein expression was analyzed by ELISA. (c) In situ hybridization of CXCL10 mRNA. Retinal frozen sections from control and IR-performed mice at $6 \mathrm{~h}$ after IR were hybridized with a probe against mouse CXCL10 and detected with RNAscope Fluorescent Multiplex Kit. Green fluorescent signal reflects CXCL10 mRNA expression and DAPI (blue) stains nuclei. Arrows indicate CXCL10-expressed retinal ganglion cells. GCL: ganglion cell layer; INL: inner nuclear layer; ONL: outer nuclear layer. (d and e) The mRNA levels of CXCL4 and CXCR3 were determined by qPCR. (f) Retinal frozen sections from control and IR-performed mice at $6 \mathrm{~h}$ after IR were incubated with an antibody against CXCR3. Fluorescent signal (red) reflects CXCR3 staining. ${ }^{*} P<0.05$ compared with control. $N=3-4$ 

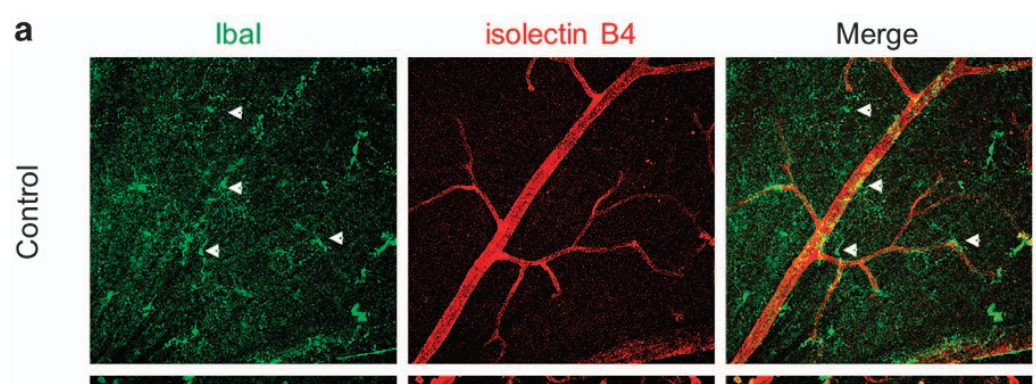

b
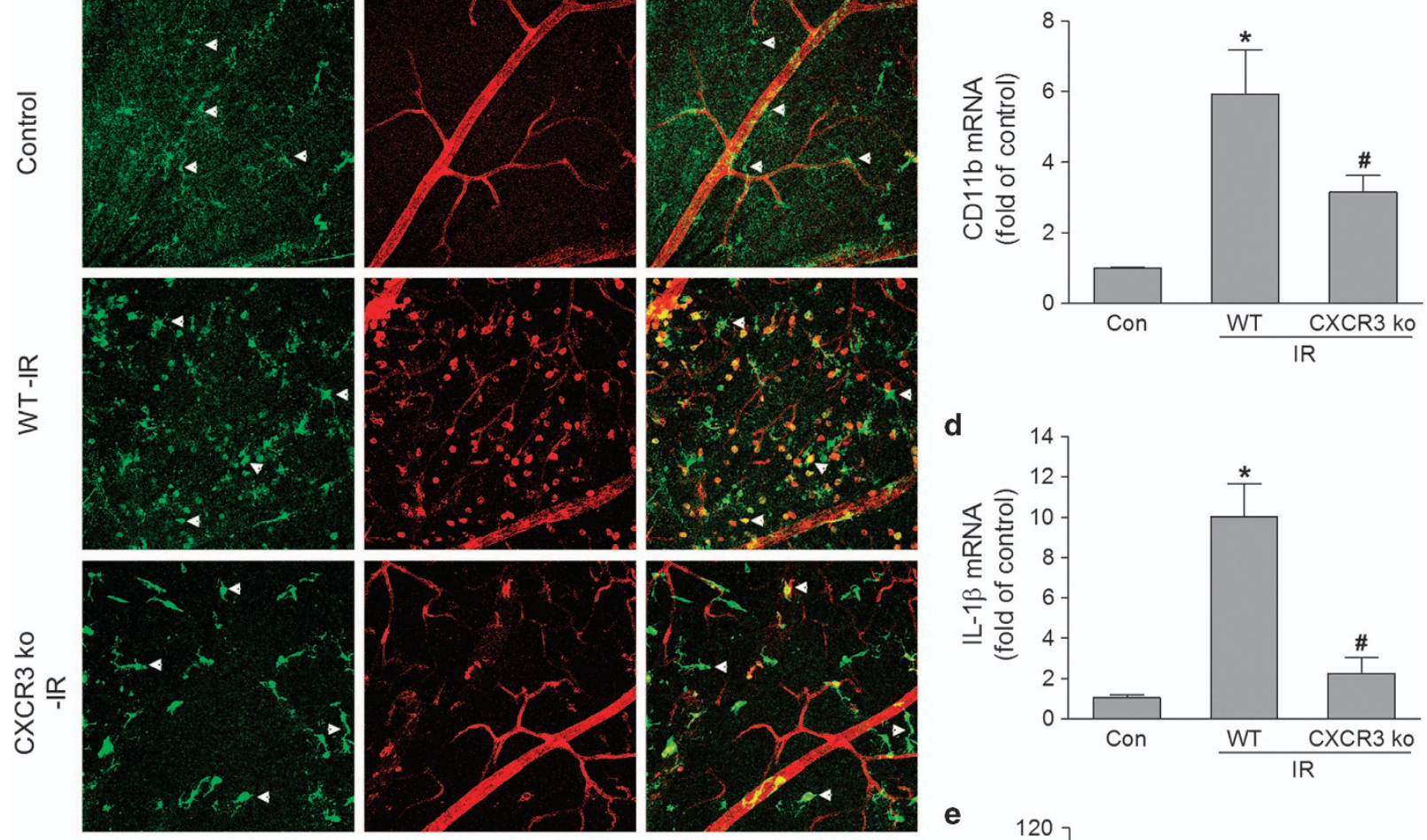

c
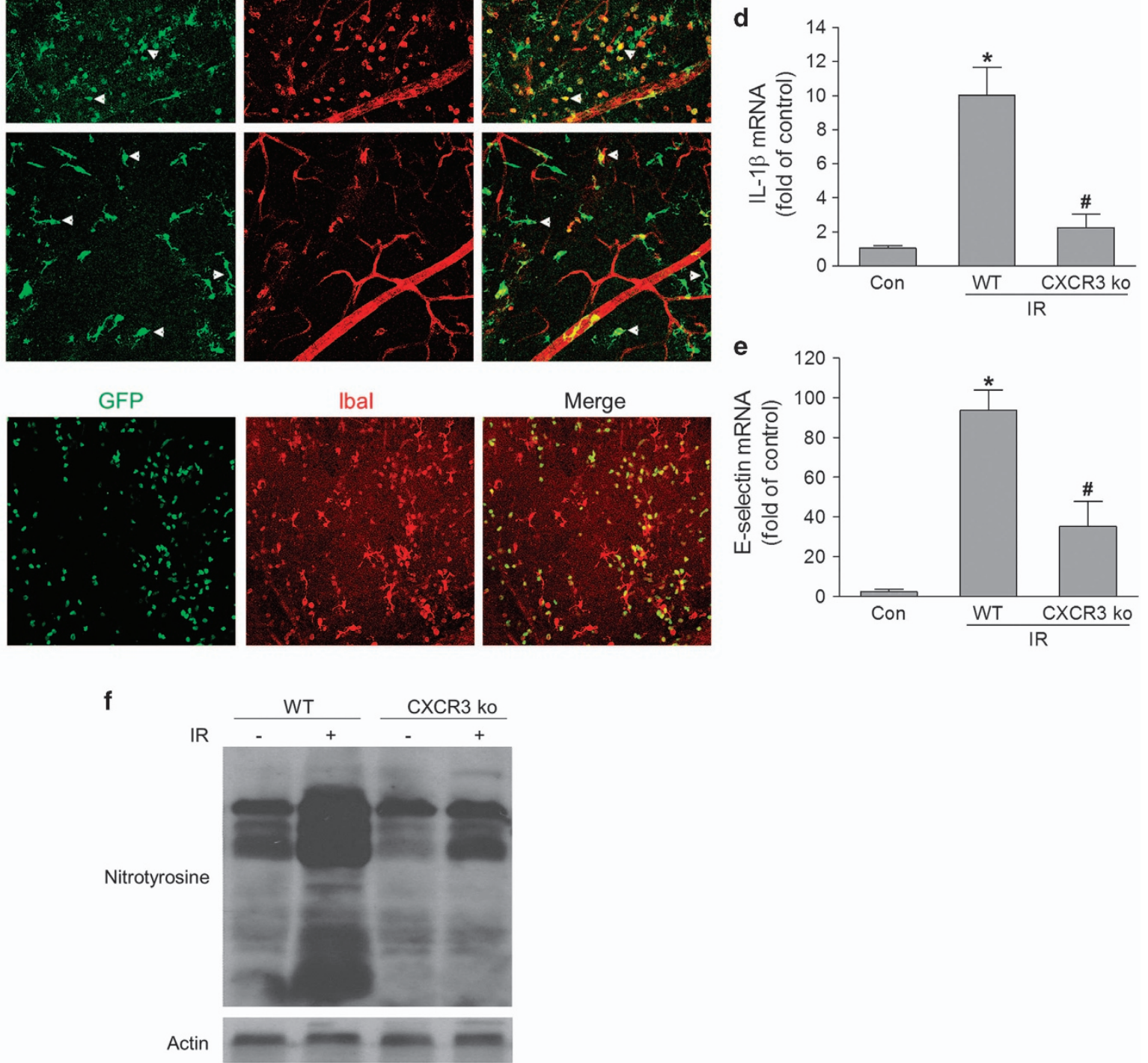

Figure 2 CXCR3 deletion reduces retinal inflammation as well as oxidative and nitrosative stress in retina after IR. Ischemia-reperfusion was induced in WT and CXCR3 ko mice and retinas were collected at $24 \mathrm{~h}$ after IR. (a) Retina was stained with isolectin B4 (red, for vessels and activated microglia/monocyte) and lbal antibody (green, for microglia/ monocyte). Arrows show microglia/monocyte with different morphology suggesting different activation status. (b) The mRNA level of CD11b in retinas was analyzed by qPCR. (c) WT mice received bone marrow transplant from GFP mice and 6 weeks later they were subjected to IR. Retinas were fixed and labeled for lbal (red) at $24 \mathrm{~h}$ after IR. (d and e) qPCR analysis of IL-1 $\beta$ and E-selectin mRNA expression in retina at $24 \mathrm{~h}$ after IR. (f) Nitrotyrosine level in retina was analyzed by western blot at $24 \mathrm{~h}$ after IR. Actin was used as an internal loading control. ${ }^{*} P<0.05$ compared with control; ${ }^{\#} P<0.05$ compared with WT-IR. $N=3-4$ 
a

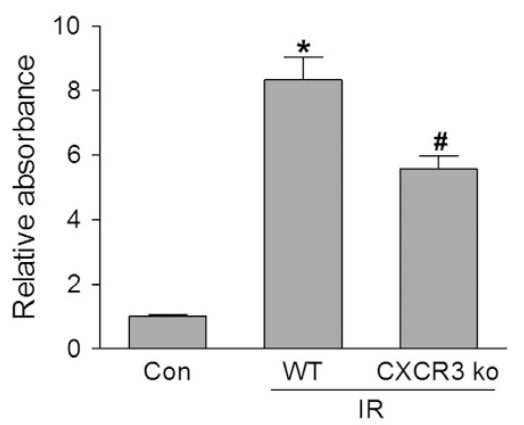

b

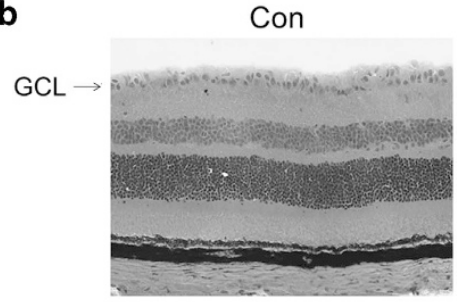

WT-IR

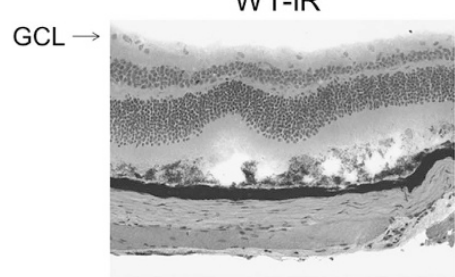

d
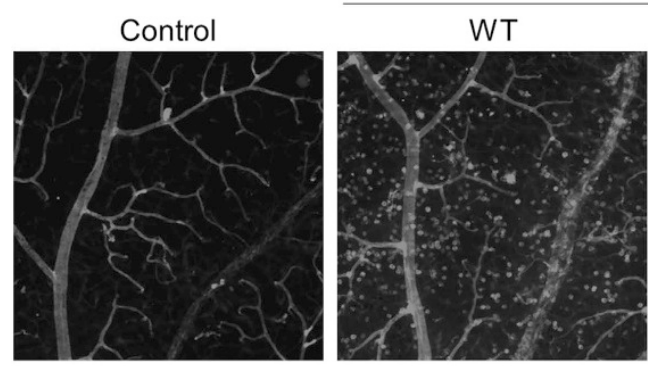

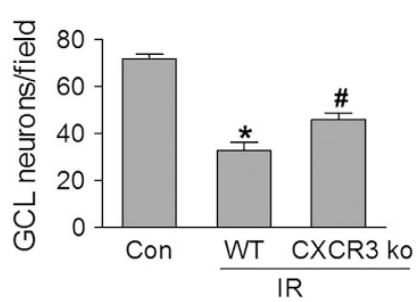

CXCR3 ko-IR

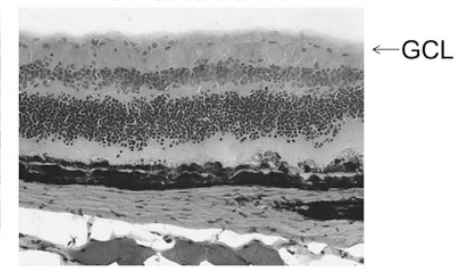

CXCL10

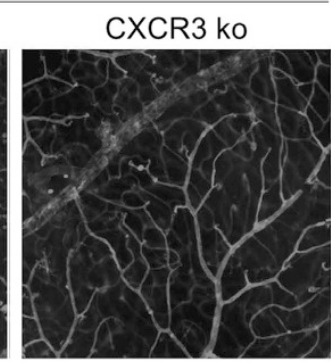

c
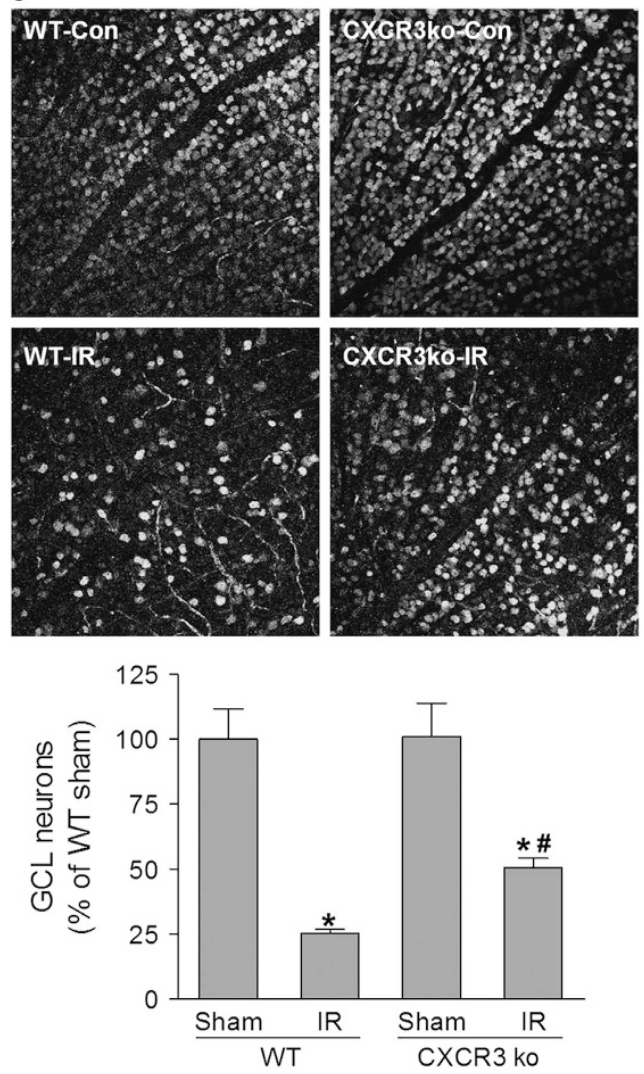

e

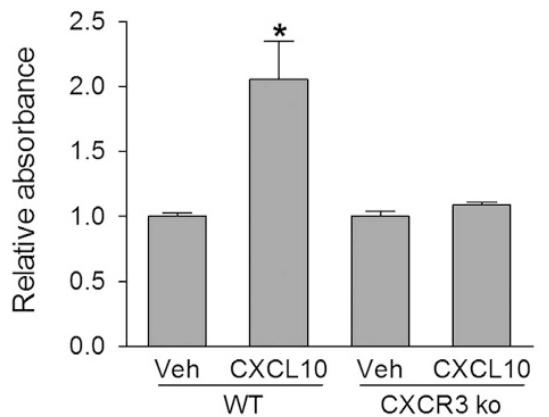

Figure 3 CXCR3 is necessary and sufficient for IR-induced GCL neuronal cell loss. WT and CXCR3 ko mice were subjected to IR. (a) Retinas were collected at $24 \mathrm{~h}$ after IR and analyzed for histone-associated DNA fragmentation to determine apoptosis. (b) Hematoxylin \& eosin (H\&E) staining of retinal section at 7 days after IR. The number of cells in ganglion cell layer (GCL) was quantified. (c) Retinas were immunostained with NeuN antibody at 7 days after IR and images were taken by confocal microscopy. NeuN-positive cells in GCL were quantified and normalized to uninjured controls. ${ }^{*} P<0.05$ compared with sham control; ${ }^{\#} P<0.05$ compared with WT-IR. (d and e) WT and CXCR3 ko mice were intravitreally injected with CXCL10 (1 $\mu$ g/eye) or vehicle (PBS) and retinas were collected after $24 \mathrm{~h}$ from injection. (d) Retinas were stained with isolectin B4 to view vessels and recruited microglia/monocyte. (e) Retinal cell apoptosis was analyzed by detecting histone-associated DNA fragmentation. ${ }^{*} P<0.05$ compared with vehicle- treated WT or CXCL10-treated CXCR3 ko mice. $N=3-4$

localized in the GCL, INL and, outer nuclear layer although were more prominent in the INL. Similarly, the number of apoptotic cells was significantly reduced in retinas from CXCR3 ko mice (Supplementary Figure S1). Consistent with alternations in cell apoptosis, H\&E-stained retinal sections showed that the neuronal cells in the GCL were strikingly decreased in WT retinas at 7 days after ischemic injury. By contrast, GCL neurons were preserved in retinas of CXCR3 ko mice (Figure $3 b$ ). This result was further confirmed by counting neuron-specific nuclear protein-positive cells within the GCL in flat-mounted retinas by confocal image analysis at 7 days and 14 days after ischemic injury (Figure $3 c$ and Supplementary Figure S2). Overall, these data demonstrated that the CXCR3 pathway is critically involved in ischemic injury-induced GCL neuronal death.

To test whether the activation of CXCR3 is sufficient to induce retinal inflammation and neuronal injury, we injected CXCL10 intravitreally and determined leukocytes recruitment and retinal cell apoptosis. As shown in Figure 3d, intravitreal delivery of CXCL10 elicited massive influx of leukocytes into 
the superficial retina in WT mice, similar to that occurred after retinal ischemic injury (Figure $2 a$ ). This effect was absent after deleting CXCR3, indicating specific CXCR3-mediated response. Associated with increases in retinal inflammatory reactions, CXCL10 treatment also induced cell death in WT retina but not in CXCR3-deficient retina (Figure 3e). Analysis of cell apoptosis by TUNEL assay revealed that CXCL10induced apoptotic cells were localized in neurons in the GCL, INL and, outer nuclear layer (Supplementary Figure S3). Taken together, these results implicate the CXCR3 ligands/ CXCR3 pathway as a key player in retinal inflammatory reactions and neuronal injury.

\section{Upregulation of CXCL10 and CXCR3 is medicated by ER} stress. Given that CXCL10 and its receptor CXCR3 are involved in retinal inflammation and neuronal injury, it would be important to understand the potential mechanisms underlying the upregulation of this pathway after retinal ischemia. The ER represents the cellular quality control site for the folding and assembly of proteins. When the function of ER is perturbed, it causes ER stress, which helps to compensate for damage. However, it may trigger cell death if the ER stress is severe. Because ER stress has key roles in inflammation in many diseases and retinal neuronal injury, ${ }^{28-31}$ we explored the potential role of $\mathrm{ER}$ stress in regulation of $\mathrm{CXCL10/}$ CXCR3 pathway. At 3 and $24 \mathrm{~h}$ after retinal ischemia, expression of ER stress markers (GRP78, CHOP, and ATF4) (Figures $4 a-c)$ was significantly increased, indicating that ER stress was elevated in our model. To determine the role and mechanism of ER stress in CXCL10 expression, we then treated mice with 4-phenylbutyric acid and tauroursodeoxycholic acid, which are chemical chaperones that are widely used to block ER stress, ${ }^{32-34}$ and assessed CXCL10 expression. Inhibiting ER stress by both 4-phenylbutyric acid and tauroursodeoxycholic acid treatment attenuated CXCL10 expression by 61 and $43 \%$, respectively, compared with untreated mice (Figure 4d). However, expression of ER stress markers were not altered by deleting CXCR3 (Figure $4 \mathrm{e}-\mathrm{g}$ ), suggesting that ER stress is not a downstream event after CXCR3 activation.

We further determined whether induction of ER stress is sufficient to induce the upregulation of CXCL10 and CXCR3 pathway. As CXCL10 mRNA and the immunoreactivity of CXCR3 were positive in GCL neurons (Figures 1c and f), we cultured mouse primary retinal ganglion cells (RGCs) and treated them with the ER stress inducer tunicamycin. The expression of ER stress markers was markedly increased after tunicamycin treatment, demonstrating successful induction of ER stress. Accordant with the upregulation of ER stress (Figures 5a-c), expression of CXCL10 and CXCR3 was increased by approximately twofold (Figures $5 d$ and e). These results indicate that ER stress serves as an upstream signaling pathway to upregulate CXCL10 and CXCR3 expression after ischemic injury.

CXCR3 pathway is involved in ER stress-induced retinal cell death. As ER stress can induce expression of CXCL10 and $\mathrm{CXCR} 3$ and the activation of CXCR3 pathway is involved in retinal neuronal cell death (Figures 3-5), we investigated whether ER stress-induced CXCL10 expression has a role in
ER stress-induced retinal neuronal injury. To do so, we injected tunicamycin intravitreally to WT and CXCR3-deficient mice. Tunicamycin induced significant increases in levels of CXCL10 and ER stress markers in retinas (Figures 6a-d) and there was no difference between WT and CXCR3-deficient mice, suggesting both genotypes respond to tunicamycin similarly. However, retinal cell apoptosis was induced threefold in retinas of WT mice compared with untreated controls, whereas it was only slightly induced in CXCR3-deficient mice (Figure 6e). These data support the notion that CXCR3 activation has an important role in ER stress-induced retinal neuronal injury.

\section{Discussion}

During acute glaucoma, increased IOP leads to retinal neuronal injury, but the mechanisms remain to be defined. Here we provide the first evidence that activation of chemokine receptor CXCR3 is critically involved in retinal inflammation and neuronal cell damage. Using a mouse model of acute glaucoma, we found that IOP-induced retinal ischemic injury increases the expression of CXCR3 as well as its two ligands CXCL10 and CXCL4 in retina. By blocking CXCR3 with gene deletion or activating CXCR3 with intravitreal injection of CXCL10, we clearly demonstrate that CXCR3 is involved in retinal inflammation, oxidative, and nitrosative stress, and retinal neuronal death during acute glaucoma. This study, together with previous studies showing that activation of CXCR3 induces damage of trabecular tissue and consequently increases IOP and our ongoing work showing CXCL10 is increased in a mouse model of open-angle glaucoma (data not shown), ${ }^{35}$ highlights the potential value of CXCR3 as a target for glaucoma therapy, as blocking CXCR3 has dual beneficial effects of protecting retinal neurons and reducing IOP. The limitation of current study is that the mouse model used has different aspects from the human acute angle closure glaucoma such as the site of putative pressure-induced damage in glaucoma, the anatomy of the optic nerve of the mouse and the higher level but shorter duration of induced IOP. ${ }^{4}$ A number of CXCR3 antagonists are in clinical development for treating diseases such as arthritis and psoriasis, ${ }^{36}$ future evaluation of the effects of these antagonists on patients with acute glaucoma would validate the role of CXCR3 in human subjects and may lead to novel therapies.

The interaction of chemokines and their receptors have a key role in guiding leukocytes migration during inflammatory reactions. In spite of having been extensively studied in inflammatory diseases in other organs, the involvement of chemokines in ocular diseases has been less studied and most of these work has focused on severe ocular inflammatory conditions such as uveitis, infectious keratitis, allergic eye diseases, and corneal transplantation. ${ }^{37}$ In retinal diseases, the role of monocyte chemoattractant protein-1 and its receptor CCR2 are well known in age-related macular degeneration, diabetic retinopathy, and photoreceptor apoptosis after retinal detachment. ${ }^{38-42}$ In addition, CCR3 polymorphism is linked to age-related macular degeneration and blocking CCR3 inhibits choroidal neovascularization in a mouse model of wet age-related macular degeneration. ${ }^{43,44}$ 


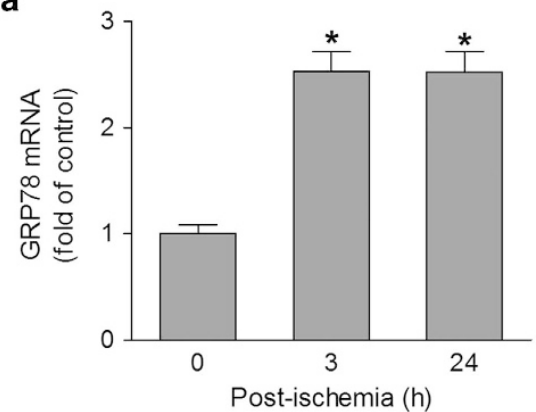

C

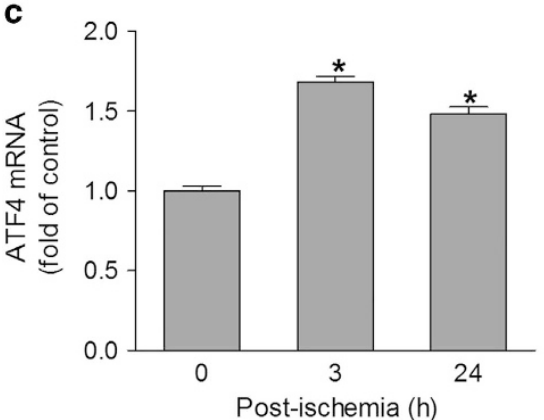

e
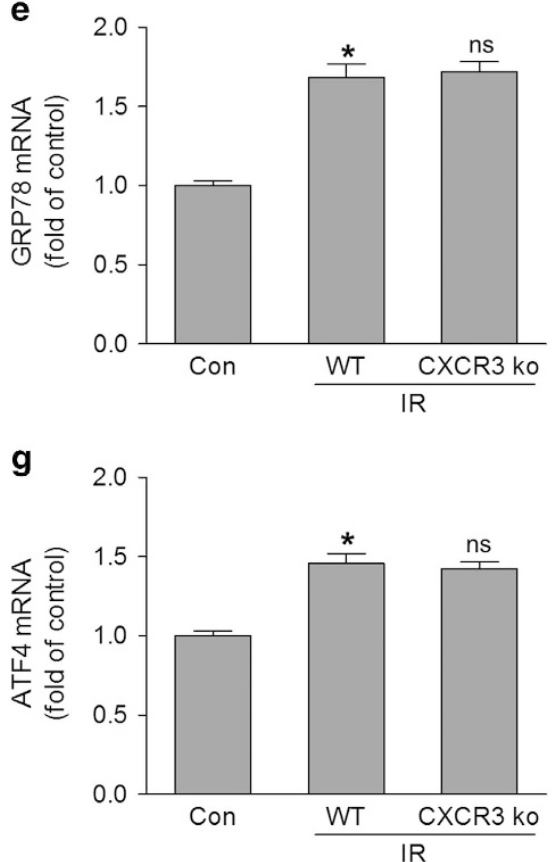

b

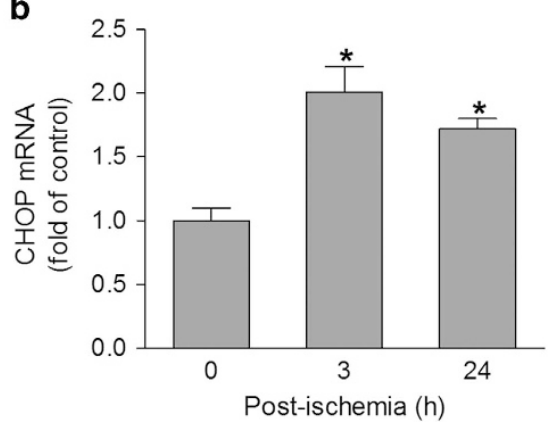

d

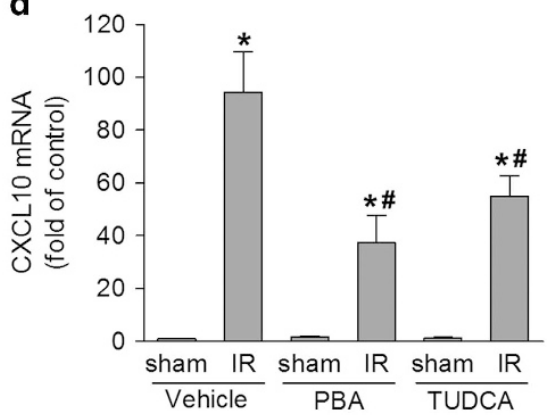

f

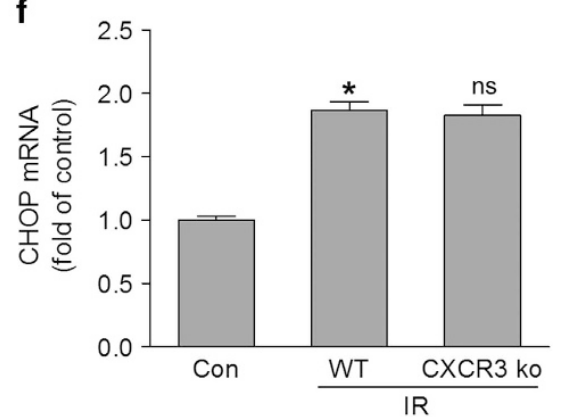

Figure 4 ER stress is involved in CXCL10 expression. (a-c) WT mice were exposed to IR and retinas were collected at 3 and $24 \mathrm{~h}$ after injury. The expression of GRP78, CHOP, and ATF4 mRNA was analyzed by qPCR. ${ }^{*} P<0.05$ compared with control. (d) WT mice were injected intraperitoneally with ER stress inhibitors, 4-phenylbutyric acid (PBA) or tauroursodeoxycholic acid (TUDCA) at $1 \mathrm{~h}$ before IR injury. Retinas were collected $6 \mathrm{~h}$ later and CXCL10 mRNA expression was assessed by qRT-PCR. ${ }^{*} P<0.05$ compared with sham control; ${ }^{\# P}<0.05$ compared with vehicle-treated IR. (e-g) Twenty-four hours after WT and CXCR3 ko mice were exposed to IR injury, retina mRNA was extracted to analyze ER stress target genes (GRP78, CHOP and ATF4) by qPCR. ${ }^{*} P<0.05$ versus control; ns: not significant compared with WT-IR. $N=4$

Our current finding that the CXCR3 pathway mediates retinal inflammation and neuronal injury in a model of acute glaucoma represents the first study that chemokines are involved in the pathogenesis of glaucomatous neuropathy. Given that chemokine receptors belong to the superfamily of $G$ protein-coupled receptors, which are the major targets for drug development and numerous specific antagonists have been developed for chemokine receptors, further investigation of chemokines in acute and chronic inflammation during retinopathies will be clinically beneficial.

At present, the precise mechanisms by which activation of CXCR3 regulates retinal neuronal injury remain to be 

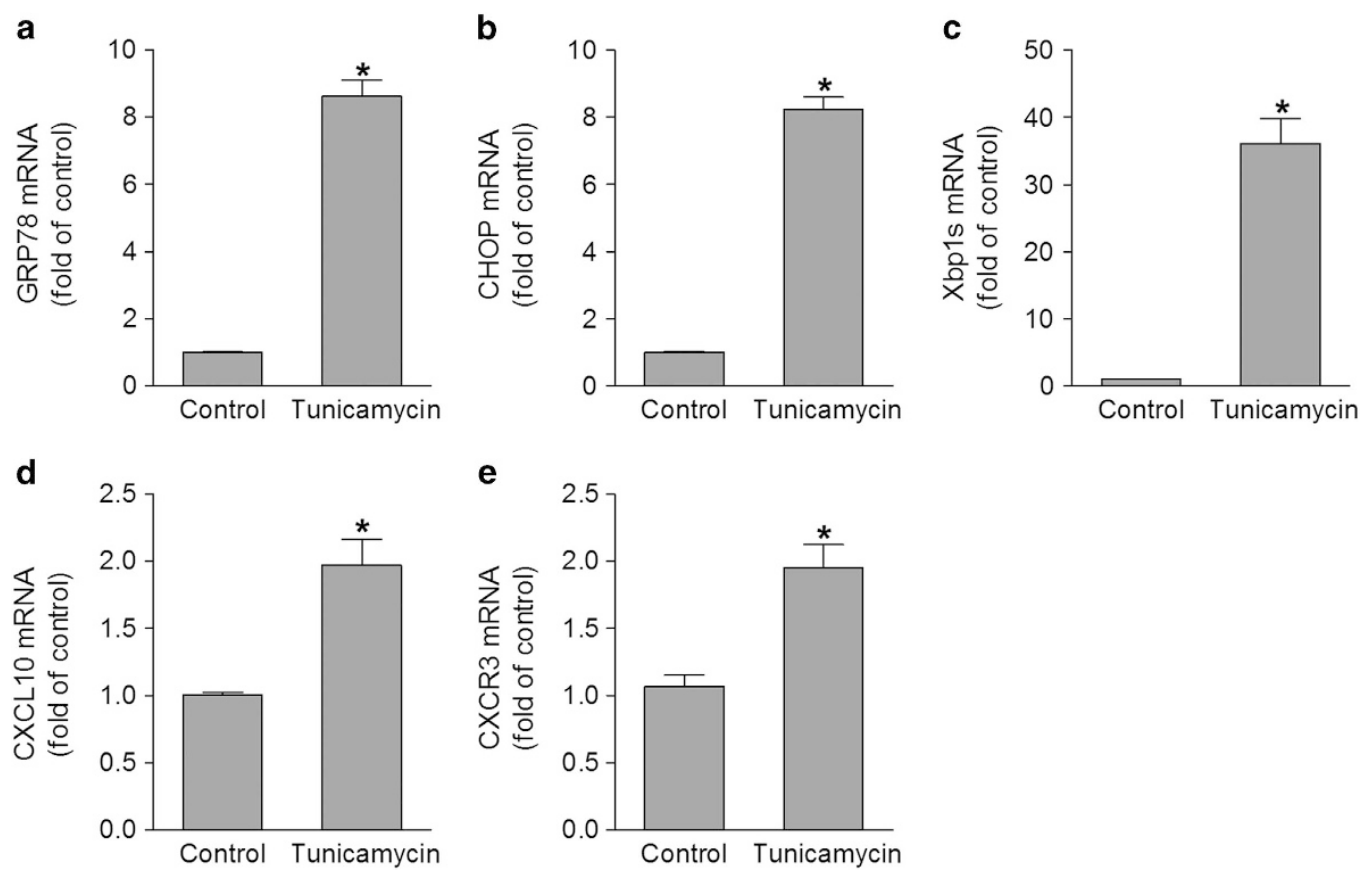

Figure 5 ER stress induction promotes $C X C L 10$ expression. Primary retinal ganglion cells were isolated and treated with $2 \mu \mathrm{g} / \mathrm{ml}$ of tunicamycin for $24 \mathrm{~h}$. The $\mathrm{mRNA}$ levels of ER stress target genes (GRP78, CHOP, and Xbp1s) (a-c), CXCL10 (d), and CXCR3 (e) were quantified by qPCR. ${ }^{*} \mathrm{P}<0.05$ compared with control. $N=4$
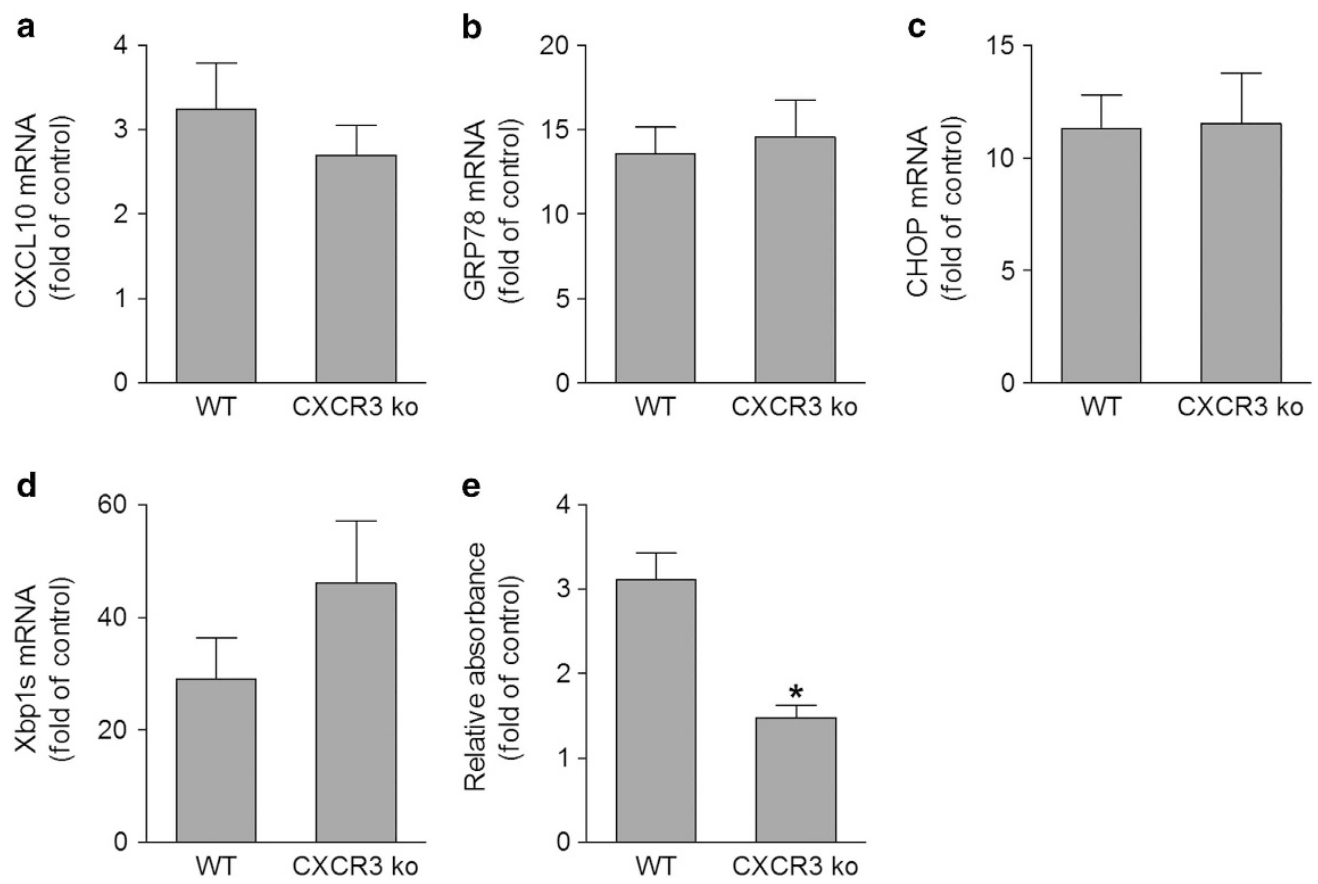

Figure 6 CXCR3 deletion attenuates ER stress-induced retinal cell apoptosis. WT and CXCR3 ko mice were intravitreally injected with tunicamycin (1 $\mu \mathrm{g} / \mathrm{eye})$ and retinas were collected at $24 \mathrm{~h}$ after injection. (a-d) qPCR was performed to measure the mRNA expression of CXCL10 and ER stress markers GRP78, CHOP, Xbp1s. (e) Apoptosis was analyzed by detecting histone-associated DNA fragmentation. ${ }^{*} P<0.05$ compared with tunicamycin-injected WT. $N=3$

elucidated. In our study, CXCR3 ligands (e.g., CXCL10) are produced in the GCL cells of retina after ischemic injury. It is possible that these ligands bind to their receptor CXCR3, which is expressed in microglia, monocytes and activated $\mathrm{T}$ cells, and recruit them to the superficial retina. After activated by CXCL10 and/or other cytokine expressed in retina, these inflammatory cells produce more cytokines and chemokines to exaggerate inflammatory cascade, resulting in increase in leukocyte recruitment and progressive inflammation. ${ }^{45,46}$ Some inflammatory cytokines produced 
by inflammatory cells further induce neuronal death by activating apoptotic signaling pathways. ${ }^{47-49}$ This possibility is supported by our data that deleting CXCR3 attenuates overall inflammatory reactions in ischemia-injured retina, including the reduction of microglia/monocyte recruitment/ activation and the decreases in expression of adhesion molecule (E-selectin) and neural toxic and pro-inflammatory cytokine (interleukin-1 $\beta$ ). In addition to neural toxic cytokines, reactive oxygen species and reactive nitrogen species generated by inflammatory cells also induce neuronal damage through a mechanism of oxidation and nitration in lipids and organelle proteins. ${ }^{50}$ Therefore, the reduction of oxidative stress and nitrosative stress by CXCR3 deletion also contributes to its neuroprotective effect. In line with this notion, deleting NOX2/NADPH oxidase, which is a major source of oxidative stress in inflammatory cells, similarly reduces peroxynitrite formation and protects neurons in the GCL. ${ }^{27}$ Overall, our data strongly suggest that CXCR3 is indirectly involved in retinal neuronal injury by recruiting inflammatory cells. However, CXCR3 signaling is reported to directly elicit apoptosis in fetal neurons. ${ }^{51}$ We demonstrate CXCR3 is markedly upregulated in GCL neurons after ischemic injury, thus we presume that CXCR3 might sensitize these neurons to CXCL10-induced apoptotic response. Further studies to specifically deleting CXCR3 in retinal neurons, microglia, and blood leukocytes are required to explore the precise mechanisms of CXCR3-induced retinal inflammation and neuronal injury in acute glaucoma. Regardless of the cell type-specific functions of CXCR3, blockade of CXCR3 with a pharmacological inhibitor may be beneficial for reducing retinal neuronal damage. Interestingly, loss of CXCR3 also prevents ER stress-induced retinal cell death, suggesting activation of the CXCR3 pathway is involved in neuronal injury in a variety of conditions and blocking this pathway is neuroprotective.

Although Ibal antibody stains both monocytes and microglia, by overlapping with GFP-positive cells, we found that Ibal-positive monocytes have different morphology from Ibal-positive microglia (Figure 2c). Infiltrated monocytes are smaller and have weaker immunoreactivity for anti-lbal antibody. In addition, monocytes display a round or suborbicular morphology, whereas microglia exhibits a dendritic morphology. By comparing the morphology of Ibal-positive cells and their infiltration patterns in CXCR3-deficient retina with those in WT retina after ischemia-reperfusion (IR) (Figure 2a), our data show that CXCR3 deletion not only reduces microglial reactivity, but also prevents the massive infiltration of peripheral monocytes into superficial retina after IR. This result suggests that the CXCR3 pathway is critically involved in regulating monocyte recruitment during retinal inflammation.

Inflammation is involved in most neurodegenerative diseases, although the triggers of the inflammatory response are poorly characterized. ${ }^{52} \mathrm{CXCL} 10$ is normally expressed at a low level under physiological conditions but is upregulated during infection or inflammation. In this study, we found that upregulation of CXCL10 is associated with increases in ER stress-related genes in ischemia-injured retina and blocking ER stress attenuates CXCL10 expression. On the other hand, the induction of ER stress promotes the expression of CXCL10 and its receptor. Although multiple cell types may express CXCL10 under stress, our study demonstrates that RGCs are one of the major sources of CXCL10 in response to ER stress. Moreover, CXCR3 expression is similarly upregulated by inducing ER stress in these cells. To our knowledge, this is the first report that ER stress regulates CXCL10 and CXCR3 levels under pathological condition. Although ER stress is able to activate NF-KB, IRF-1, and IRF-3, and these transcription factors regulate $\mathrm{CXCL} 10$ expression in response to different stimuli, ${ }^{28,53-56}$ the specific mediators linking ER stress to CXCL10 and CXCR3 expression in acute glaucoma remain to be elucidated.

In summary, our data reveal a novel mechanism that ER stress-regulated activation of CXCR3 pathway contributes to retinal inflammation and neuronal injury during acute glaucoma. As CXCL10 is also upregulated in other diseases associated with neuronal injury, such as diabetic retinopathy and stroke, ${ }^{57,58}$ our study warrants further investigation of the CXCL10/CXCR3 axis in these diseases to determine whether or not this pathway has a general role in inducing inflammation and neuronal death and therefore targeting this pathway could be beneficial in a variety of diseases.

\section{Materials and Methods}

Animals. The experimental procedures and use of animals were performed in accordance with the Association of Research for Vision and Ophthalmology Statement for the Use of Animals in Ophthalmic and Vision Research, and all protocols were approved by the Institutional Animal Care and Use Committee at the University of Texas Medical Branch. C57BL/6 J, CXCR3KO and GFP transgenic mice were obtained from Jackson Laboratory (Bar Harbor, ME, USA) and maintained on a 12:12 light/dark cycle with food and water available ad libitum.

Ischemia-reperfusion. Mice (8-12 weeks) were anesthetized with a mixture of ketamine hydrochloride $(100 \mathrm{mg} / \mathrm{kg})$ and xylazine hydrochloride $(10 \mathrm{mg} / \mathrm{kg})$ by intraperitoneal injection and $0.5 \%$ proparacine hydrochloride was administered topically. The anterior chamber of the right eye was cannulated with a 30-gauge infusion needle connected to a saline reservoir. IOP was raised to $110 \mathrm{~mm} \mathrm{Hg}$ for $45 \mathrm{~min}$ by elevating the saline reservoir. ${ }^{4,27} \mathrm{~A}$ sham procedure performed without elevating the pressure in the left eye served as the control. Eyes and retinas were collected from $3 \mathrm{~h}$ to 14 days after IR for further analysis. To investigate the involvement of ER stress in retinal pathogenesis, ER stress inhibitors, 4-phenylbutyric acid and tauroursodeoxycholic acid (Sigma-Aldrich, St Louis, MO, USA) were injected (i.p., $500 \mathrm{mg} / \mathrm{kg}$ ) $1 \mathrm{~h}$ before IR was performed.

Intravitreal injection. Intravitreal injection was performed as described previously. ${ }^{59} 1 \mu \mathrm{l}$ PBS containing $1 \mu \mathrm{g}$ of mouse CXCL10 (PeproTech, Rocky Hill, $\mathrm{NJ}$, USA) was delivered by a 35-gauge needle. PBS was subjected as control to the contralateral eye of the same animal. After $24 \mathrm{~h}$ of injection, eyes and retinas were collected for further analysis.

Bone marrow transplantation. Bone marrow transplantation was performed as described previous. ${ }^{60}$ In brief, WT mice at 6 weeks of age underwent whole-body gamma irradiation with $137 \mathrm{Cs}$ using a Gammacell 40 irradiator (MDS Nordion, Ontario, Canada). A lead shield was used to protect the head and eyes from radiation. A dose of $8.5 \mathrm{~Gy}$ (850 rads) was used to ablate the marrow. Within $24 \mathrm{~h}$ after irradiation, mice received bone marrow transplant from 6-week-old GFP transgenic mice through tail vein as a $200 \mu \mathrm{l}$ cell suspension containing $0.7-1.0 \times 10^{7}$ cells. 6 weeks later, mice were subjected to IR.

Isolation of primary RGC. Primary RGCs were isolated from WT mouse pups at postnatal day 3 . Detailed procedures have been described in the previous study. ${ }^{61,62}$ In brief, collected retinas were dissociated in a papain solution $(15 \mathrm{U} / \mathrm{ml})$ and incubated with anti-macrophage antiserum to remove the macrophages and microglial cells. Non-adherent cells were then incubated with mouse Thy-1.2 antibody (BD Biosciences, San Diego, CA, USA) to isolate ganglion cells. Cells were seeded at a density of $2.3 \times 10^{5} \mathrm{cells} /$ well and incubated at $37^{\circ} \mathrm{C}$. The purity of 
the RGCs was determined by staining with mouse Tuj1 antibody (BioLegend, San Diego, CA, USA), a specific RGC marker. To determine whether tunicamycin could induce the expression of CXCL10, cells were exposed to $2 \mu \mathrm{g} / \mathrm{ml}$ tunicamycin for $24 \mathrm{~h}$.

Real time quantitative RT-PCR analysis and primers used. Total RNA was isolated using RNAqueous-4PCR kit (Life Technologies, Carlsbad, CA, USA) according to the manufacturer's instruction. CDNA was generated by reverse transcription using High Capacity cDNA Reverse Transcription Kit (Life Technologies). Quantitative PCR was performed using a StepOnePlus PCR system (Life Technologies) with Power SYBR Green (Life Technologies). The fold difference in various transcripts was calculated by the $\triangle \Delta C T$ method using Hprt as the internal control. Primer sequences for mouse transcripts were as follows: Hprt For-5'-GAA AGA CTT GCT CGA GAT GTC ATG-3'; Hprt Rev-5'-CAC ACA GAG GGC CAC AAT GT-3'; CXCL10 For-5'-CAT CCC TGC GAG CCT ATC C-3'; CXCL10 Rev-5'-CAT CTC TGC TCA TCA TTC TTT TTC A-3'; CXCL4 For-5'-CGG TTC CCC AGC TCA TAG C-3'; CXCL4 Rev-5'-CCG GTC CAG GCA AAT TTT C-3'; CXCR3 For-5'-TTG CCC TCC CAG ATT TCA TC-3'; CXCR3 Rev-5'-TGG CAT TGA GGC GCT GAT-3'; GRP78 For-5'-ACT TGG GGA CCA CCT ATT CCT-3'; GRP78 Rev-5'-ATC GCC AAT CAG ACG CTC C-3'; ATF4 For-5'-TCC TGA ACA GCG AAG TGT TG-3'; ATF4 Rev-5'ACCC ATGAGGTTTCAAGTGC-3'; CHOP For-5'-CTG GAA GCC TGG TAT GAG GAT-3'; CHOP Rev-5'-CAG GGT CAA GAG TAG TGA AGG T-3'; Xbp1s For-5'-TGC TGA GTC CGC AGC AGG TG-3'; Xbp1s Rev-5'- GCT GGC AGG CTC TGG GGA AG-3'.

Western blot. Proteins were isolated from neuronal retina and subjected to SDS-PAGE. PVDF membranes, to which the proteins had been transferred, were incubated with primary antibody against nitrotyrosine (1:500; Cayman Chemical, Ann Arbor, MI, USA), followed with HRP-conjugated goat anti-mouse secondary antibody (1:2000; Amersham Biosciences, Piscataway, NJ, USA). Proteins were detected using the enhanced chemiluminescence system (Pierce, Rockford, IL, USA). The membranes were reprobed with monoclonal antibody against $\beta$-actin (1:5000; Sigma-Aldrich) as a loading control.

In Situ hybridization. Retinal frozen sections were incubated with mouse CXCL10 probe (Advanced Cell Diagnostics, Hayward, CA, USA) and sequentially hybridized to a cascade of signal amplification molecules from RNAscope Fluorescent Multiplex detection reagent kit (Advanced Cell Diagnostics) according to the manufacturer's instructions. At last, slides were counterstained with DAPI to label nuclei and viewed by epifluorescence microscope to detect CXCL10 mRNApositive cells.

Immunohistochemical analysis. Retinal frozen sections were fixed in $4 \%$ paraformaldehyde in PBS, washed, and blocked with PowerBlock (Biogenx, San Ramon, CA, USA) for $1 \mathrm{~h}$. Subsequently, sections were incubated with primary antibody against CXCR3 $(1: 250 ;$ R\&D Systems, Minneapolis, MN, USA). After washing, retinal sections were incubated with Alexa Fluor 594-labeled goat antirabbit secondary antibody (1:1000; Life Technologies). Coverslips were mounted on slides with Fluoroshield with DAPI histology mounting medium (Sigma-Aldrich) and sections were examined by an Olympus 1X71 epifluorescence microscope.

Immunostaining of retinal whole mounts. After the fixation in $4 \%$ paraformaldehyde, retinas were dissected from choroid and sclera, blocked and permeabilized in PBS containing 5\% normal goat serum and 0.3\% Triton-X-100 for $1 \mathrm{~h}$. Subsequently, retinas were stained with Alexa Fluor 594-labeled isolectin B4 (Griffonia simplicifolia) (1:200; Life Technologies) and antibody against neuronspecific nuclear protein $(1: 400$; Millipore, Billerica, MA, USA) or lba1 $(1: 400$; Wako, Osaka, Japan) overnight at $4^{\circ} \mathrm{C}$. Retinas were then incubated with Alexa Fluor 488-conjugated donkey anti-mouse or Alexa Fluor 488-labeled goat anti-rabbit secondary antibody (1:400). After washing with PBS, retinas were mounted on a microscope glass slide using Vectashield mounting medium (Vector Laboratories, Burlingame, CA, USA), and examined by confocal microscopy.

ELISA. CXCL10 concentration in ischemia-reperfused retina was determined using Quantikine Mouse CXCL10 Immunoassay Kit (R\&D Systems) following the manufacturer's instructions. The optical density was measured using Synergy $\mathrm{H} 1$ microplate reader (BioTek, Winooski, VT, USA).
Cell death detection ELISA. To detect the apoptotic cell death in the ischemic injured retinas of WT or CXCR3 ko mice, a cell death detection ELISA ${ }^{\text {PLUS }}$ kit (Roche Molecular Biochemicals, Indianapolis, IN, USA) was used to quantify DNA fragmentation according to the manufacturer's instructions. The optical density was measured using Synergy $\mathrm{H} 1$ microplate reader.

The TUNEL assay. To visualize apoptotic cells, TUNEL assay was performed on retinal frozen sections with ApopTag Fluorescein in situ Apoptosis Detection Kit (Millipore) according to the manufacturer's instructions. Retinal sections were counterstained with DAPI to label nuclei and TUNEL-positive cells were counted under epifluorescence microscope.

Statistical analysis. Data were presented as Mmean \pm S.E.M. Results were analyzed using GraphPad Prism (GraphPad Software Inc., La Jolla, CA, USA). Comparison between experimental groups was made by Student's $t$-test and oneway ANOVA followed by post hoc Student's t-test using the Student-Newman-Keuls method. A value of $P<0.05$ was considered statistically significant. Data shown are representative of three or more independent experiments.

\section{Conflict of Interest}

The authors declare no conflict of interest.

Acknowledgements. This work was supported by National Institutes of Health grant EY022694, American Heart Association 11SDG4960005, a grant from International Retinal Research Foundation, and the John Sealy Memorial Endowment Fund for Biomedical Research (to WZ); by National Institutes of Health grants EY11766 (to RBC and RWC), EY 14560 (to SBS) and VA Merit Award (to RBC); and by American Heart Association 15POST22450025 (to HL).

1. Ang LP, Ang LP. Current understanding of the treatment and outcome of acute primary angle-closure glaucoma: an Asian perspective. Ann Acad Med Singapore 2008; 37 : 210-215.

2. Weinreb RN, Aung T, Medeiros FA. The pathophysiology and treatment of glaucoma: a review. JAMA 2014; 311: 1901-1911.

3. Quek DT, Koh VT, Tan GS, Perera SA, Wong TT, Aung T. Blindness and long-term progression of visual field defects in chinese patients with primary angle-closure glaucoma. Am J Ophthalmol 2011; 152: 463-469.

4. Chi W, Li F, Chen H, Wang Y, Zhu Y, Yang X et al. Caspase-8 promotes NLRP1/NLRP3 inflammasome activation and IL-1beta production in acute glaucoma. Proc Natl Acad Sci USA 2014; 111: 11181-11186.

5. Zhang W, Liu H, Al-Shabrawey M, Caldwell RW, Caldwell RB. Inflammation and diabetic retinal microvascular complications. J Cardiovasc Dis Res 2011; 2: 96-103.

6. Urrutia PJ, Mena NP, Nunez MT. The interplay between iron accumulation, mitochondrial dysfunction, and inflammation during the execution step of neurodegenerative disorders. Front Pharmacol 2014; 5: 38.

7. Wax MB, Tezel G. Immunoregulation of retinal ganglion cell fate in glaucoma. Exp Eye Res 2009; 88: 825-830.

8. Howell GR, Soto I, Zhu X, Ryan M, Macalinao DG, Sousa GL et al. Radiation treatment inhibits monocyte entry into the optic nerve head and prevents neuronal damage in a mouse model of glaucoma. J Clin Invest 2012; 122: 1246-1261.

9. Dvoriantchikova G, Barakat DJ, Hernandez E, Shestopalov VI, Ivanov D. Toll-like receptor 4 contributes to retinal ischemia/reperfusion injury. Mol Vis 2010; 16: 1907-1912.

10. Lacotte S, Brun S, Muller S, CXCR3 Dumortier H. Inflammation, and autoimmune diseases. Ann N Y Acad Sci 2009; 1173: 310-317.

11. Mueller A, Meiser A, McDonagh EM, Fox JM, Petit SJ, Xanthou G et al. CXCL4-induced migration of activated $T$ lymphocytes is mediated by the chemokine receptor CXCR3. J Leukoc Biol 2008; 83: 875-882.

12. Pervushina O, Scheuerer B, Reiling N, Behnke L, Schroder JM, Kasper B et al. Platelet factor $4 / C X C L 4$ induces phagocytosis and the generation of reactive oxygen metabolites in mononuclear phagocytes independently of $\mathrm{Gi}$ protein activation or intracellular calcium transients. J Immunol 2004; 173: 2060-2067.

13. Srivastava K, Cockburn IA, Swaim A, Thompson LE, Tripathi A, Fletcher CA et al. Platelet factor 4 mediates inflammation in experimental cerebral malaria. Cell Host Microbe 2008; 4: 179-187.

14. de Jong EK, de Haas AH, Brouwer N, van Weering HR, Hensens M, Bechmann I et al. Expression of CXCL4 in microglia in vitro and in vivo and its possible signaling through CXCR3. J Neurochem 2008; 105: 1726-1736.

15. Taub DD, Lloyd AR, Conlon K, Wang JM, Ortaldo JR, Harada A et al. Recombinant human interferon-inducible protein 10 is a chemoattractant for human monocytes and T lymphocytes and promotes T cell adhesion to endothelial cells. $J$ Exp Med 1993; 177 : 1809-1814. 
16. Torraca V, Cui C, Boland R, Bebelman JP, van der Sar AM, Smit MJ et al. The CXCR3CXCL11 signaling axis mediates macrophage recruitment and dissemination of mycobacterial infection. Dis Model Mech 2015; 8: 253-269.

17. Wang Y, Gehringer R, Mousa SA, Hackel D, Brack A, Rittner HL. CXCL10 controls inflammatory pain via opioid peptide-containing macrophages in electroacupuncture. PLOS One 2014; 9: e94696.

18. Wang X, Ellison JA, Siren AL, Lysko PG, Yue TL, Barone FC et al. Prolonged expression of interferon-inducible protein-10 in ischemic cortex after permanent occlusion of the middle cerebral artery in rat. J Neurochem 1998; 71: 1194-1204.

19. Galimberti D, Schoonenboom N, Scheltens P, Fenoglio C, Bouwman F, Venturelli E et al. Intrathecal chemokine synthesis in mild cognitive impairment and Alzheimer disease. Arch Neurol 2006; 63: 538-543

20. Balashov KE, Rottman JB, Weiner HL, Hancock WW. CCR5(+) and CXCR3(+) T cells are increased in multiple sclerosis and their ligands MIP-1alpha and IP-10 are expressed in demyelinating brain lesions. Proc Natl Acad Sci USA 1999; 96: 6873-6878.

21. Jo N, Wu GS, Rao NA. Upregulation of chemokine expression in the retinal vasculature in ischemia-reperfusion injury. Invest Ophthalmol Vis Sci 2003; 44: 4054-4060.

22. Rappert A, Bechmann I, Pivneva T, Mahlo J, Biber K, Nolte $C$ et al. CXCR3-dependent microglial recruitment is essential for dendrite loss after brain lesion. $J$ Neurosci 2004; 24 8500-8509.

23. van Weering HR, Boddeke HW, Vinet J, Brouwer $\mathrm{N}$, de Haas $\mathrm{AH}$, van Rooijen $\mathrm{N}$ et al CXCL10/CXCR3 signaling in glia cells differentially affects NMDA-induced cell death in CA and DG neurons of the mouse hippocampus. Hippocampus 2011; 21: 220-232.

24. Rappert A, Biber K, Nolte C, Lipp M, Schubel A, Lu B et al. Secondary lymphoid tissue chemokine (CCL21) activates CXCR3 to trigger a $\mathrm{Cl}$ - current and chemotaxis in murine microglia. J Immunol 2002; 168: 3221-3226.

25. Dommergues MA, Plaisant F, Verney C, Gressens P. Early microglial activation following neonatal excitotoxic brain damage in mice: a potential target for neuroprotection. Neuroscience 2003; 121: 619-628.

26. Zarbock A, Ley K, McEver RP, Hidalgo A. Leukocyte ligands for endothelial selectins: specialized glycoconjugates that mediate rolling and signaling under flow. Blood 2011; 118 6743-6751.

27. Yokota H, Narayanan SP, Zhang W, Liu H, Rojas M, Xu Z et al. Neuroprotection from retinal ischemia/reperfusion injury by NOX2 NADPH oxidase deletion. Invest Ophthalmol Vis Sci 2011; 52: 8123-8131.

28. Garg AD, Kaczmarek A, Krysko O, Vandenabeele P, Krysko DV, Agostinis P. ER stressinduced inflammation: does it aid or impede disease progression? Trends Mol Med 2012; 18 : 589-598.

29. Shimazawa M, Inokuchi Y, Ito Y, Murata H, Aihara M, Miura M et al. Involvement of ER stress in retinal cell death. Mol Vis 2007; 13: 578-587.

30. Jeng YY, Lin NT, Chang PH, Huang YP, Pang VF, Liu CH et al. Retinal ischemic injury rescued by sodium 4-phenylbutyrate in a rat model. Exp Eye Res 2007; 84: 486-492.

31. Hu Y, Park KK, Yang L, Wei X, Yang Q, Cho KS et al. Differential effects of unfolded protein response pathways on axon injury-induced death of retinal ganglion cells. Neuron 2012; 73 : 445-452.

32. Ozcan U, Yilmaz E, Ozcan L, Furuhashi M, Vaillancourt E, Smith RO et al. Chemica chaperones reduce ER stress and restore glucose homeostasis in a mouse model of type 2 diabetes. Science 2006; 313: 1137-1140.

33. Kawasaki N, Asada R, Saito A, Kanemoto S, Imaizumi K. Obesity-induced endoplasmic reticulum stress causes chronic inflammation in adipose tissue. Sci Rep 2012; 2: 799.

34. Ishimura S, Furuhashi M, Mita T, Fuseya T, Watanabe $\mathrm{Y}$, Hoshina K et al. Reduction of endoplasmic reticulum stress inhibits neointima formation after vascular injury. Sci Rep 2014; 4: 6943.

35. Denoyer A, Godefroy D, Celerier I, Frugier J, Degardin J, Harrison JK et al. CXCR3 antagonism of SDF-1(5-67) restores trabecular function and prevents retinal neurodegeneration in a rat model of ocular hypertension. PLoS One 2012; 7: e37873.

36. Michlmayr D, Lim JK. Chemokine receptors as important regulators of pathogenesis during arboviral encephalitis. Front Cell Neurosci 2014; 8: 264

37. Wallace GR, John Curnow S, Wloka K, Salmon M, Murray PI. The role of chemokines and their receptors in ocular disease. Prog Retin Eye Res 2004; 23: 435-448.

38. Rangasamy S, McGuire PG, Franco Nitta C, Monickaraj F, Oruganti SR, Das A. Chemokine mediated monocyte trafficking into the retina: role of inflammation in alteration of the bloodretinal barrier in diabetic retinopathy. PLOS One 2014; 9: e108508.

39. Zhang W, Rojas M, Lilly B, Tsai NT, Lemtalsi T, Liou Gl et al. NAD(P)H oxidase-dependent regulation of CCL2 production during retinal inflammation. Invest Ophthalmol Vis Sci 2009; 50: $3033-3040$

40. Anand A, Sharma NK, Gupta A, Prabhakar S, Sharma SK, Singh R et al. Single nucleotide polymorphisms in MCP-1 and its receptor are associated with the risk of age related macular degeneration. PLoS One 2012; 7: e49905.
41. Ambati J, Anand A, Fernandez S, Sakurai E, Lynn BC, Kuziel WA et al. An animal model of age-related macular degeneration in senescent Ccl-2- or Ccr-2-deficient mice. Nat Med 2003; 9: 1390-1397.

42. Nakazawa T, Hisatomi T, Nakazawa $\mathrm{C}$, Noda K, Maruyama K, She $\mathrm{H}$ et al. Monocyte chemoattractant protein 1 mediates retinal detachment-induced photoreceptor apoptosis. Proc Natl Acad Sci USA 2007; 104: 2425-2430.

43. Sharma NK, Gupta A, Prabhakar S, Singh R, Bhatt AK, Anand A. CC chemokine receptor-3 as new target for age-related macular degeneration. Gene 2013; 523: 106-111.

44. Takeda A, Baffi JZ, Kleinman ME, Cho WG, Nozaki M, Yamada K et al. CCR3 is a target for age-related macular degeneration diagnosis and therapy. Nature 2009; 460: 225-230.

45. Mennicken F, Maki R, de Souza EB, Quirion R. Chemokines and chemokine receptors in the CNS: a possible role in neuroinflammation and patterning. Trends Pharmacol Sci 1999; 20: 73-78.

46. Andersson PB, Perry VH, Gordon S. Intracerebral injection of proinflammatory cytokines or leukocyte chemotaxins induces minimal myelomonocytic cell recruitment to the parenchyma of the central nervous system. J Exp Med 1992; 176: 255-259.

47. Banati RB, Gehrmann J, Schubert P, Kreutzberg GW. Cytotoxicity of microglia. Glia 1993; 7 : 111-118.

48. Barone FC, Arvin B, White RF, Miller A, Webb CL, Willette RN et al. Tumor necrosis factoralpha. A mediator of focal ischemic brain injury. Stroke 1997; 28: 1233-1244.

49. Wang XJ, Kong KM, Qi WL, Ye WL, Song PS. Interleukin-1 beta induction of neuron apoptosis depends on p38 mitogen-activated protein kinase activity after spinal cord injury. Acta Pharmacol Sin 2005; 26: 934-942.

50. Loh KP, Huang SH, De Silva R, Tan BK, Zhu YZ. Oxidative stress: apoptosis in neuronal injury. Curr Alzheimer Res 2006; 3: 327-337.

51. Sui Y, Stehno-Bittel L, Li S, Loganathan R, Dhillon NK, Pinson D et al. CXCL10-induced cell death in neurons: role of calcium dysregulation. Eur J Neurosci 2006; 23: 957-964.

52. Tansey MG, McCoy MK, Frank-Cannon TC. Neuroinflammatory mechanisms in Parkinson's disease: potential environmental triggers, pathways, and targets for early therapeutic intervention. Exp Neurol 2007; 208: 1-25.

53. Wang YI, Bettaieb A, Sun C, DeVerse JS, Radecke CE, Mathew S et al. Triglyceride-rich lipoprotein modulates endothelial vascular cell adhesion molecule (VCAM)-1 expression via differential regulation of endoplasmic reticulum stress. PLOS One 2013; 8: e78322.

54. Zaheer RS, Proud D. Human rhinovirus-induced epithelial production of CXCL10 is dependent upon IFN regulatory factor-1. Am J Respir Cell Mol Biol 2010; 43: 413-421.

55. Brownell J, Bruckner J, Wagoner J, Thomas E, Loo YM, Gale M et al. Direct, InterferonIndependent Activation of the CXCL10 Promoter by NF-kappa B and Interferon Regulatory Factor 3 during Hepatitis C Virus Infection. J Virol 2014; 88: 1582-1590.

56. Liu YP, Zeng L, Tian A, Bomkamp A, Rivera D, Gutman D et al. Endoplasmic reticulum stress regulates the innate immunity critical transcription factor IRF3. J Immunol 2012; 189: 4630-4639.

57. Wang X, Li X, Schmidt DB, Foley JJ, Barone FC, Ames RS et al. Identification and molecular characterization of rat CXCR3: receptor expression and interferon-inducible protein-10 binding are increased in focal stroke. Mol Pharmacol 2000; 57: 1190-1198.

58. Maier R, Weger M, Haller-Schober EM, El-Shabrawi Y, Wedrich A, Theisl A et al. Multiplex bead analysis of vitreous and serum concentrations of inflammatory and proangiogenic factors in diabetic patients. Mol Vis 2008; 14: 637-643.

59. Ameri H, Liu H, Liu R, Ha Y, Paulucci-Holthauzen AA, Hu S et al. TWEAK/Fn14 pathway is a novel mediator of retinal neovascularization. Invest Ophthalmol Vis Sci 2014; 55: 801-813.

60. Rojas M, Zhang W, Xu Z, Lemtalsi T, Chandler P, Toque HA et al. Requirement of NOX2 expression in both retina and bone marrow for diabetes-induced retinal vascular injury. PLoS One 2013; 8: e84357.

61. Ha Y, Dun Y, Thangaraju M, Duplantier J, Dong Z, Liu K et al. Sigma receptor 1 modulates endoplasmic reticulum stress in retinal neurons. Invest Ophthalmol Vis Sci 2011; 52: $527-540$

62. Ha Y, Saul A, Tawfik A, Zorrilla EP, Ganapathy V, Smith SB. Diabetes accelerates retinal ganglion cell dysfunction in mice lacking sigma receptor 1. Mol Vis 2012; 18: 2860-2870.

Cell Death and Disease is an open-access journal published by Nature Publishing Group. This work is licensed under a Creative Commons Attribution 4.0 International License. The images or other third party material in this article are included in the article's Creative Commons license, unless indicated otherwise in the credit line; if the material is not included under the Creative Commons license, users will need to obtain permission from the license holder to reproduce the material. To view a copy of this license, visit http://creativecommons.org/licenses/by/4.0/ 